\title{
Spin-Orbit Coupling in Gyrotropic Quantum Wells by Far-Infrared Radiation Induced Spin-Galvanic Effect
}

\author{
S.D. Ganichev, V.V Bel'kov*, Petra Schneider, S. Giglberger, Ch. Hoffmann, and W. Prettl \\ Universität Regensburg, D 93040 Regensburg, Germany
}

\begin{abstract}
The Rashba effect, whose experimental access is usually masked by the Dresselhaus effect, allows manipulation of spins in semiconductor spintronics. Based on the far-infrared radiation induced spingalvanic effect, we present a unique way to separate both types of spin-orbit coupling.
\end{abstract}

\section{Introduction}

The manipulation of the spin of charge carriers in semiconductors is one of the key problems in the field of spintronics. In the paradigmatic spin transistor, e.g. proposed by Datta and Das [1], the electron spins injected from a ferromagnetic contact into a twodimensional electron system are controllably rotated during their passage from source to drain by means of the Rashba spin-orbit coupling [2]. The coefficient $\alpha$, which describes the strength of the Rashba spinorbit coupling, and hence the degree of rotation, can be tuned by gate voltages. This coupling stems from the inversion asymmetry of the confining potential of two-dimensional electron (or hole) systems. In addition to the Rashba coupling, caused by structure inversion asymmetry (SIA), also a Dresselhaus type of coupling of strength $\beta$ contributes to the spin-orbit interaction. The latter is due to bulk inversion asymmetry (BIA) including phenomenologically inseparable interface inversion asymmetry (IIA) [3]. Both, Rashba and Dresselhaus couplings result in spin-splitting of subbands in $\boldsymbol{k}$-space (Fig. 1) and give rise to a variety of spin dependent phenomena.

However, usually it is impossible to extract the relative contributions of Rashba and Dresselhaus terms to the spin-orbit coupling. To obtain the Rashba coefficient $\alpha$, the Dresselhaus contribution is normally neglected. Here we show that angular dependent measurements of the spin-galvanic photocurrent in the far-infrared [4] allow to separate contributions due to Dresselhaus and Rashba terms. We make use of the fact that these terms contribute differently for particular crystallographic directions.

\section{Spin-orbit interaction}

We consider quantum wells (QWs) of zinc-blende structure grown in [001] direction. For the corresponding $\mathrm{C}_{2 v}$ symmetry the spin-orbit part $\hat{H}_{S O}$ of the Hamiltonian
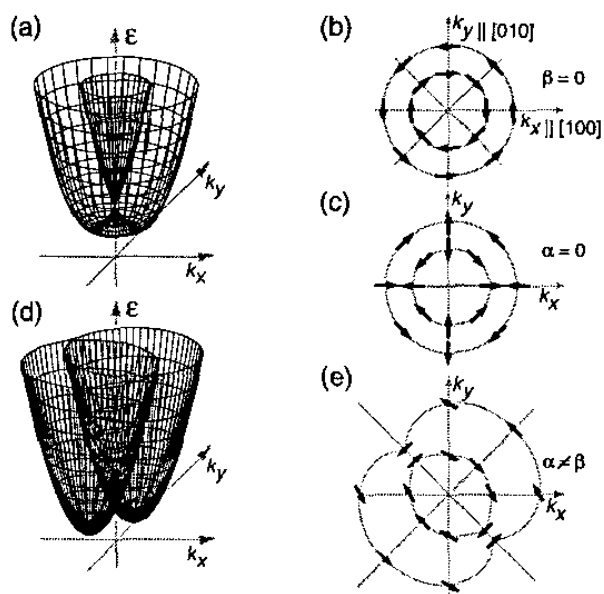

Figure 1: Schematic 2D band structure with $k$-linear terms for $\mathrm{C}_{2 v}$ symmetry for different relative strengths of SIA and BIA and the distribution of spin orientations at the 2D Fermi energy: (a) shows the case of only Rashba or Dresselhaus spin-orbit coupling and (d) represents the case of simultaneous presence of both contributions. Arrows indicate the orientation of spins.

$\hat{H}=\hbar^{2} k^{2} / 2 m^{*}+\hat{H}_{S O}$ contains the Rashba term as well as the Dresselhaus term according to

$$
\hat{H}_{S O}=\alpha\left(\sigma_{x} k_{y}-\sigma_{y} k_{x}\right)+\beta\left(\sigma_{x} k_{x}-\sigma_{y} k_{y}\right)
$$

where $k$ is the electron wavevector and $\sigma$ is the vector of the Pauli matrices. The $x$-axis is aligned along the [100]-direction, $y$ along [010], and $z$ is the growth direction.

The resulting energy dispersion $\varepsilon(k)$ and contours of constant energy in the $k_{x}, k_{y}$ plane for different $\alpha$ and $\beta$ are illustrated in Fig. 1. For $\alpha \neq 0, \beta=0$ and $\alpha=0$, $\beta \neq 0$ the dispersion has the same shape and consists of two parabolas shifted in all directions (Fig. 1a). However, Rashba and Dresselhaus terms result in a different pattern of the eigenstate's spin orientation. The distribution of this spin orientation is obtained by diagonalizing $\hat{H}_{S O}$ in Eq. (1). In the presence of both Rashba and Dresselhaus spin-orbit couplings, relevant for $\mathrm{C}_{2 v}$ symmetry, the [110] and the [110] axes become strongly non-equivalent yielding an anisotropic dispersion sketched in Fig. 1d,e. 


\section{Experimental}

The experiments were carried out at room temperature on (001)-oriented $n$-type InAs $/ \mathrm{Al}_{0.3} \mathrm{Ga}_{0.7} \mathrm{Sb}$ single QWs of $15 \mathrm{~nm}$ width having $C_{2 v}$ point symmetry. The free carrier densities and mobilities at room temperature were about $1.3 \cdot 10^{12} \mathrm{~cm}^{-2}$ and $\approx 2 \cdot 10^{4} \mathrm{~cm}^{2} /(\mathrm{Vs})$, respectively. Eight opposite pairs of contacts on each sample allow to probe the photocurrent in different directions (see Fig. 2b). For optical spin orientation we use a high power pulsed molecular far-infrared $\mathrm{NH}_{3}$ laser at $\lambda=148 \mu \mathrm{m}$. Circular polarization of the linearly polarized emission is obtained by a $\lambda / 4$ quartz plate.

The photocurrent $j_{S G E}$ is measured in unbiased structures via the voltage drop across a $50 \Omega$ load resistor in a closed circuit configuration [5]. It is detected for right $\left(\sigma_{+}\right)$and left $\left(\sigma_{-}\right)$handed circularly polarized radiation. The spin-galvanic current $j_{S G E}$, studied here, is extracted after eliminating current contributions which are helicity independent: $j_{S G E}=\left(j_{\sigma_{+}}-j_{\sigma_{-}}\right) / 2$.

\section{Results and Discussion}

We employ the spin-galvanic effect to extract the ratio of the Rashba and the Dresselhaus contributions. The spin-galvanic current is driven by the electron in-plane average spin $S_{\|}$according to $[4,5]$ :

$$
j_{S G E} \propto\left(\begin{array}{cc}
\beta & -\alpha \\
\alpha & -\beta
\end{array}\right) \boldsymbol{S}_{\|}
$$

Therefore, the spin-galvanic current $j_{S G E}$ for a certain direction of $S_{||}$consists of Rashba and Dresselhaus coupling induced currents, $j_{R}$ and $j_{D}$ (see Fig. 2a). Their magnitudes are $j_{R} \propto \alpha\left|S_{\|}\right|, j_{D} \propto \beta\left|S_{\|}\right|$and their ratio is

$$
j_{R} / j_{D}=\alpha / \beta .
$$

The non-equilibrium in-plane spin polarization $\boldsymbol{S}_{\|}$is prepared as described recently [4]: Circularly polarized light at normal incidence on the $\mathrm{QW}$ plane polarizes the electrons in the lowest conduction subband resulting in a monopolar spin orientation in the $z$-direction (Fig. 2b). An in-plane magnetic field $(B=1 \mathrm{~T})$ rotates the spin around the magnetic field axis (precession) and results in a non-equilibrium in-plane spin polarization $S_{\|} \propto \omega_{L} \tau_{s}$, where $\omega_{L}$ is the Larmor frequency and $\tau_{s}$ is the spin relaxation time. In the range of the applied magnetic field strength the spin-galvanic current in the present samples at room temperature rises linearly with $B$ indicating $\omega_{L} \tau_{s}<1$ and, thus, the Hanle effect is not present. The angle between the magnetic field and $S_{\mid l}$ in general depends on details of the spin relaxation process. In the InAs QW structures investigated here, the isotropic Elliott-Yafet spin relaxation mechanism dominates [6]. Thus the in-plane spin polarization $\boldsymbol{S}_{\|}$ of photoexcited carriers is always perpendicular to $B$ and can be varied by rotating $\boldsymbol{B}$ around $z$ as illustrated

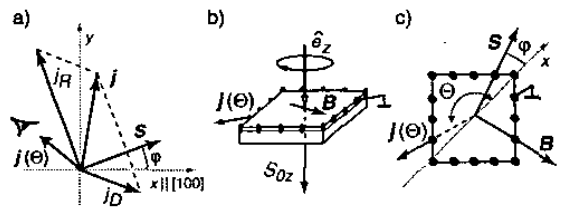

Figure 2: Angular dependence of the spin-galvanic current (a) and geometry of the experiment (b and c).

in Fig. 2c. This excess spin polarization $S_{\|}$leads to an increase of the population of the corresponding spinpolarized states. Due to asymmetric spin relaxation an electric current results [4].

To obtain the Rashba- and Dresselhaus contributions the spin-galvanic effect is measured for a fixed orientation of $S_{\|}$for all accessible directions $\Theta$ (see Fig. 2c). According to Eq. (2) the current $j_{R}$ always flows perpendicularly to the spin polarization $S_{\|}$, and $j_{D}$ encloses an angle $-2 \varphi$ with $S_{\|}$. Here $\varphi$ is the angle between $S_{\|}$and the $x$-axis. Then, the current component along any direction given by angle $\Theta$ can be written as a sum of the projections of $j_{R}$ and $j_{D}$ on this direction

$$
j_{S G E}(\Theta)=j_{D} \cos (\Theta+\varphi)+j_{R} \sin (\Theta-\varphi) .
$$

Evaluating the measurements using this equation yields immediately the ratio between Rashba and Dresselhaus terms. The best value obtained here is $j_{R} / j_{D}=\alpha / \beta=$ 2.1 in a good agreement to theoretical results [7] which predict a dominating Rashba spin-orbit coupling for InAs QWs.

Financial support by the DFG is gratefully acknowledged.

${ }^{*}$ Permanent address: A.F. Ioffe Physico-Technical Institute, 194021 St. Petersburg, Russia

\section{References}

[1] S. Datta, and B. Das, Appl. Phys. Lett. 56,665 (1990).

[2] Y.A. Bychkov, and E.I. Rashba, Pis'ma ZhETF 39, 66 (1984) [Sov. JETP Lett. 39, 78 (1984)].

[3] U. Rössler, and J. Kainz, Solid State Commun. 121, 313 (2002).

[4] S.D. Ganichev, E.L. Ivchenko, V.V. Bel'kov, S.A. Tarasenko, M. Sollinger, D. Weiss, W. Wegscheider, and W. Prettl, Nature (London) 417, 153 (2002).

[5] S.D. Ganichev, and W. Prettl, J. Phys.: Condens. Matter 15, R935 (2003), and references cited therein.

[6] N.S. Averkiev, L.E. Golub, and M. Willander, J. Phys.: Condens. Matter 14, R271 (2002).

[7] G. Lommer, F. Malcher, and U. Rössler, Phys. Rev. Lett. 60, 728 (1988). 